\title{
A Solution of the 4th Clay Millennium Problem about the Navier-Stokes Equations
}

\author{
Konstantinos E. Kyritsis
}

\begin{abstract}
In this paper it is solved the $4^{\text {th }}$ Clay Millennium problem about the Navier-Stokes equations, in the direction of regularity (no blow-up). This is proved for the Navier-Stokes equations for the non-periodic formulation and without external forcing (homogeneous case).The proof is based on discovering a new invariant as a 2D surface density of (rotatory) momentum, derived from the well-known Helmholtz-Kelvin-Stokes velocity circulation invariant. This invariant is indispensable, besides to the ordinary momentum conservation, to prove that there cannot be a blow-up in finite time, of the point vorticities, thus regularity..It is proved that not only there is no Blow-up in finite time but not even at the time $\mathbf{T}=+\infty$.
\end{abstract}

Index Terms-Incompressible flows, regularity, Navier-Stokes equations, 4th Clay millennium problem. Mathematical Subject Classification: 76A02

\section{INTRODUCTION}

The famous $4^{\text {th }}$ Millennium problem of the Clay Mathematical Institute as formulated in [19] FEFFERMAN C. L. 2006 CL 2006 is considered a significant challenge to the science of mathematical physics of fluids, not only because it has resisted the efforts of the scientific community for decades to prove it (or converses to it) but also because it is supposed to hide a significant missing perception about the nature of our mathematical formulations of physical flows through the Euler and Navier-Stokes equations.

When the 4th Clay Millennium Problem was formulated in the standard way, the majority was hoping that the regularity was also valid in 3 dimensions as it had been proven to hold in 2 dimensions.

The main objective of this paper is to prove the regularity of the Navier-Stokes equations with initial data as in the standard formulation of the $4^{\text {th }}$ Clay Millennium Problem for the homogeneous (no external forcing) non-periodic case. (see paragraph IV) It is proved that not only there is no Blow-up in finite time but not even at the time $\mathbf{T}=+\infty$. My first attempt to solve the millennium problem about the regularity of the Navier-Stokes equations problem was during the spring 2013 (uploaded at that time see [25] Kyritsis K. October 2013

The author has also solved the $3^{\text {rd }}$ Millennium problem $\mathrm{P}$ vs $\mathrm{NP}$ in computational complexity with 3 different successive solutions each one simpler that the previous. (see references [29])

The main core of the solution is the paragraphs 3.In this paragraph 3 is discussed what is that probably we do not understand with the Navier-Stokes equations, and the well-known Helmholtz-Kelvin-Stokes theorem of velocity circulation is extended to new momentum density invariants of the flow. Based on these new invariants we are able to prove in theorem 3.4 that the vorticity cannot blow up (thus regularity) The paragraph 2 is devoted to reviewing the standard formulation of the $4^{\text {th }}$ Clay Millennium problem, while the $4^{\text {th }}$ paragraph simply applies trivially the results of the paragraph 3 to solve the 4th Clay Millennium problem in the non-periodic homogeneous case (no external forcing).

According to[8] CONSTANTIN P. 2007 “..The blow-up problem for the Euler equations is a major open problem of PDE, theory of far greater physical importance that the blow-up problem of the Navier-Stokes equation, which is of course known to non-specialists because of the Clay Millennium problem..." For this reason, many of the propositions of this paper are stated for the Euler equations of inviscid flows as well.

\section{THE STANDARD FORMULATION OF THE $4^{\mathrm{TH}}$ MILLENNIUM CONJECTURE ABOUT THE NAVIER-STOKES EQUATIONS AND SOME CRITERIA OF REGULARITY.}

In this paragraph we highlight the basic parts of the standard formulation of the $4^{\text {th }}$ Clay millennium problem as in [19] Fefferman C.L. 2006.

The Navier-Stokes equations are given by (by $\mathrm{R}$ we denote the field of the real numbers, $v>0$ is the density normalized viscosity coefficient )

$$
\begin{aligned}
& \frac{\partial}{\partial t} u_{i}+\sum_{j=1}^{n} u_{j} \frac{\partial u_{i}}{\partial x_{j}}=-\frac{\partial p}{\partial x_{i}}+v \Delta u_{i} \quad\left(\mathrm{x} \in \mathrm{R}^{3}, \mathrm{t}>=0\right. \\
& n=3 \text { ) } \\
& \operatorname{div} u=\sum_{i=1}^{n} \frac{\partial u_{i}}{\partial x_{i}}=0\left(\mathrm{x} \in \mathrm{R}^{3}, \mathrm{t}>=0, n=3\right)(\text { eq.2.2) } \\
& \text { a) with initial conditions } u(x, 0)=u^{0}(x) \\
& x \in R^{3} \\
& \begin{array}{l}
\text { b) and } u^{0}(x) \in C^{\infty} \text { divergence-free vector field on } \\
R^{3}
\end{array}
\end{aligned}
$$

If $v=0$ then we are taking about the Euler equations and inviscid case. 
c) $\Delta=\sum_{i=1}^{n} \frac{\partial^{2}}{\partial x_{i}^{2}}$ is the Laplacian operator.The

Euler equations are (eq2.1), (eq2.2), (eq2.3) when $v=0$.

It is reminded to the reader, that in the equations of Navier-Stokes, as in (eq. 2.1) the density $\rho$, is constant, it is custom to normalized to 1 and omit it.

d) For physically meaningful solutions we want to make sure that $u^{0}(x)$ does not grow large as $|x| \rightarrow \infty$. This is set by defining $u^{0}(x)$, and $f(x, t)$ and called in this paper Schwartz initial conditions, to satify

$\left|\partial_{x}^{a} u^{0}(x)\right| \leq C_{a, K}(1+|x|)^{-K}$ on $\mathbf{R}^{3}$ for any $\alpha$ and $\mathrm{K}$

(eq.2.4)

(Schwartz used such functions to define the space of Schwartz distributions)

Remark 2.1. It is important to realize that smooth Schwartz initial velocities after

(eq 2.4) will give that the initial vorticity $\omega_{0}=\operatorname{curl}\left(u^{0}\right)$, in its supremum norm, is bounded over all 3-space.

$\left|\partial_{x}^{a} \partial_{t}^{m} f(x, t)\right| \leq C_{a, m, K}(1+|x|+t)^{-K} \quad$ on $\quad \mathbf{R}^{3} \quad \times$ $[\mathbf{0},+\infty)$ for any $\alpha, m, \mathrm{~K} \quad$ (eq. 2.5 )

We accept as physical meaningful solutions only if it satisfies $\mathrm{p}, \mathrm{u} \in \mathrm{C}^{\infty}\left(\mathrm{R}^{3} \times[0, \infty)\right)$ (eq.2.6)

and

$\int_{\mathfrak{R}^{3}}|u(x, t)|^{2} d x<C$ for all $\mathrm{t}>=0$ (Bounded or finite energy)

Remark 2.2 It is important to realize that smooth external force (densities) with the Schwartz property as in (eq.2.5), have not only a rule for upper bounded spatial partial derivatives but also the same rule for time upper bounded partial derivatives.

Remark 2.3 We must stress here that imposing smoothness of the coordinate functions of velocities and external forces of the initial $\mathrm{t}=0$ data and later time $\mathrm{t}$ data in the Cartesian coordinates plus and Schwartz condition as in (eq 2.5) is not equivalent with imposing similar such smoothness of the coordinate functions and conditions in the cylindrical or spherical coordinates. We will give in the paragraph 4, remark 4.5 an example of a strange blowup, where at any time $\mathrm{t}>0$ the coordinates of the velocities are smooth and bounded in all space as functions in the polar coordinates and still the vorticity has infinite singularity at zero.

Alternatively, to rule out problems at infinity, we may look for spatially periodic solutions of (2.1), (2.2), (2.3). Thus we assume that $\mathrm{u}^{0}(\mathrm{x})$, and $\mathrm{f}(\mathrm{x}, \mathrm{t})$ satisfy

$u^{0}\left(x+e_{j}\right)=u^{0}(x), f\left(x+e_{j}, t\right)=f(x, t), p\left(x+e_{j}, 0\right)=p(x, 0)$,

for

$1<=\mathrm{j}<=3$ (eq. 2.8 )

$\left(e_{j}\right.$ is the $j$ th unit vector in $R^{3}$ )

In place of (2.4) and (2.5), we assume that $u^{0}(x)$, is smooth and that

$\left|\partial_{x}^{a} \partial_{t}^{m} f(x, t)\right| \leq C_{a, m, K}(1+t)^{-K}$ on $\mathbf{R}^{3} \times[\mathbf{0},+\infty)$ for any $\alpha, m, \mathrm{~K}$ (eq.2.9)

We then accept a solution of (2.1), (2.2), (2.3) as physically reasonable if it satisfies

$\mathrm{u}\left(\mathrm{x}+\mathrm{e}_{\mathrm{j}, \mathrm{t}}\right)=\mathrm{u}(\mathrm{x}, \mathrm{t}), \mathrm{p}\left(\mathrm{x}+\mathrm{e}_{\mathrm{j}}, \mathrm{t}\right)=\mathrm{p}(\mathrm{x}, \mathrm{t})$, on $\mathbf{R}^{3} \times[\mathbf{0},+\infty)$ for $1<=\mathrm{j}<=3$

and $\mathrm{p}, \mathrm{u} \in \mathrm{C}^{\infty}\left(\mathrm{R}^{3} \times[0, \infty)\right)$

(eq.2.11)
In the next paragraphs we may also write $\mathrm{u}_{0}$ instead of $\mathrm{u}^{0}$ for the initial data velocity.

We denote Euclidean balls by $B(a, r):=\{x \in$ $\left.R^{3}:\|x-a\| \leq r\right\}$, where $\|\mathrm{x}\|$ is the Euclidean norm.

The 4 sub-problems or conjectures of the millennium problem are the next:

(Conjecture A) Existence and smoothness of Navier-Stokes solution on $\mathbf{R}^{\mathbf{3}}$.

Take $v>0$ and $n=3$. Let $u_{0}(x)$ be any smooth, divergent-free vector field satisfying (4). Take $f(x, t)$ to be identically zero. Then there exist smooth functions $p(x, t), u(x, t)$ on $R^{3} x[0,+\infty)$ that satisfy (2.1), (2.2), (2.3), (2.6), (2.7).

(Conjecture B) Existence and smoothness of Navier-Stokes solution on $\mathbf{R}^{\mathbf{3}} / \mathbf{Z}^{\mathbf{3}}$.

Take $v>0$ and $n=3$. Let $u_{0}(x)$ be any smooth, divergent-free vector field satisfying (8); we take $f(x, t)$ to be identically zero. Then there exist smooth functions $p(x, t), u(x, t)$ on $R^{3} x[0,+\infty)$ that satisfy (2.1), (2.2), (2.3), (2.10), (2.11).

\section{(Conjecture C) Breakdown of Navier-Stokes solution on} $\mathbf{R}^{3}$

Take $v>0$ and $n=3$. Then there exist a smooth, divergent-free vector field $u_{0}(x)$ on $R^{3}$ and a smooth $f(x, t)$ on $R^{3} x[0,+\infty)$ satisfying (4), (5) for which there exist no smooth solution $(p(x, t), u(x, t))$ of (2.1), (2.2), (2.3), (2.6) , (2.7) on $R^{3} x[0,+\infty)$.

(Conjecture D) Breakdown of Navier-Stokes solution on $\mathbf{R}^{\mathbf{3}} / \mathbf{Z}^{\mathbf{3}}$

Take $v>0$ and $n=3$. Then there exist a smooth, divergent-free vector field $u_{0}(x)$ on $R^{3}$ and a smooth $f(x, t)$ on $R^{3} x[0,+\infty)$ satisfying (2.8), (2.9) for which there exist no smooth solution $(p(x, t), u(x, t))$ of (2.1), (2.2), (2.3), (2.10), (2.11) on $R^{3} x[0,+\infty)$.

Remark 2.4. It is stated in the same formal formulation of the Clay millennium problem by C. L. Fefferman see [19] Fefferman C.L. 2006 (see page 2nd line 5 from below) that the conjecture (A) has been proved to holds locally. "..if the time internal $[0, \infty)$, is replaced by a small time interval $[0, T)$, with $\mathrm{T}$ depending on the initial data....". In other words there is $\infty>\mathrm{T}>0$, such that there exists a unique and smooth solution $u(x, t) \in C^{\infty}\left(R^{3} \times[0, T)\right)$. See also [34] A.J. Majda-A.L. Bertozzi ,Theorem 3.4 pp 104. In this paper, as it is standard almost everywhere, the term smooth refers to the space $C^{\infty}$ In the next the \|\|$_{\mathrm{m}}$ is the corresponding Sobolev spaces norm and .We denote by $\mathrm{V}^{\mathrm{m}}=\left\{\mathrm{u}\right.$ in $\mathrm{H}^{\mathrm{m}}\left(\mathrm{R}^{\mathrm{n}}\right)$ and divu $\left.=0\right\}$ where $\mathrm{H}^{\mathrm{m}}\left(\mathrm{R}^{\mathrm{n}}\right)$ are the Sobolev spaces with the $\mathrm{L}^{2}$ norm.

We must mention that in A.J. Majda-A.L. Bertozzi [34] ,Theorem 3.4 pp 104, Local in Time existence of Solutions to the Euler and Navier-Stokes equations it is proved that indeed if the initial velocities belong to $\mathrm{V}^{\mathrm{m}} \mathrm{m}>=[3 / 2]+2$ there exist unique smooth solutions locally in time $[0, t]$. Here, in the formulation of the millennium problem the hypotheses of smooth with Schwartz condition initial velocities satisfies this condition therefore we have the existence and uniqueness of smooth solution locally in time, both in the non-periodic and the periodic setting without external forcing (homogeneous case).

The existence and uniqueness of a smooth solutions locally in time is stated in the formulation by C.L. Fefferman [19] for the homogeneous cases and conjectures (A), (B). When a smooth Schwartz condition external force is added (inhomogeneous case), it is natural to expect that also there should exist a local in time unique sooth solution. But this I 
did not find to be stated in the A.J. Majda-A.L. Bertozzi [34], so I will avoid assuming it.

We state here also two, very well-known criteria of no blow-up and regularity.

In this theorem the \|\|$_{m}$ is the corresponding Sobolev spaces norm and .We denote by $\mathrm{V}^{\mathrm{m}}=\left\{\mathrm{u}\right.$ in $\mathrm{H}^{\mathrm{m}}\left(\mathrm{R}^{\mathrm{n}}\right)$ and divu $\left.=0\right\}$ where $\mathrm{H}^{\mathrm{m}}\left(\mathrm{R}^{\mathrm{n}}\right)$ are the Sobolev spaces with the $\mathrm{L}^{2}$ norm.

\section{Theorem 2.1 Velocities Sobolev norm sufficient condition} of regularity. Given an initial condition $u_{0} \in V^{m}$ $m>=[3 / 2]+2=3.5$ e.g. $m=4$, then for any viscosity $v>=0$. there exists a maximal time $T^{*}$ (possibly infinite) of existence of a unique smooth solution $u \in C\left(\left[0, T^{*}\right] ; V^{m}\right)$ ก $C^{l}\left(\left[0, T^{*}\right] ; V^{m-2}\right)$ to the Euler or Navier-Stokes equation. Moreover, if $T^{*}<+\infty$ then necessarily $\lim _{t->T^{*}}\|u(., t)\|_{m}$ $=+\infty$.

Proof: See A.J. Majda-A.L. Bertozzi [34], Corollary 3.2 pp 112). QED

Remark 2.5 Obviously this proposition covers the periodic case too.

Theorem 2.2 Supremum of vorticity sufficient condition of regularity

Let the initial velocity $u_{0} \in V^{m} m>=[3 / 2]+2$, e.g. $m=4$, so that there exists a classical solution $u \in C^{l}\left([0, T] ; C^{2} \cap V^{m}\right)$ to the $3 D$ Euler or Navier-Stokes equations. Then:

(i) If for any $T>0$ there is $M_{1}>0$ such that the vorticity $\omega=\operatorname{curl}(u)$ satisfies

$\int_{0}^{T}|\omega(., \tau)|_{L^{\infty}} d \tau \leq M_{1}$

Then the solution $u$ exists globally in time, $u \in C^{l}([0,+\infty]$; $\left.C^{2} \cap V^{m}\right)$

(ii) If the maximal time $T^{*}$ of the existence of the solution $u \in$ $C^{l}\left([0, T] ; C^{2} \cap V^{m}\right)$ is finite,

then necessarily the vorticity accumulates so rapidly that

$\lim _{t \rightarrow T *} \int_{0}^{T}|\omega(., \tau)|_{L^{\infty}} d \tau=+\infty$

Proof: See A.J. Majda-A.L. Bertozzi [34], Theorem 3.6 pp $115, \mathrm{~L}^{\infty}$ vorticity control of regularity.

QED.

Remark 2.6 Obviously this proposition covers the periodic case too.

\section{SOME NEW MOMENTUM DENSITY INVARIANTS OF THE NAVIER-STOKES EQUATIONS AFTER THE HELMHOLTZ-KELVIN-STOKES THEOREM.}

It has been written in the initial formulation of the problem, that our difficulty of solving this millennium problem shows that there are several things that we do not understand very well in the Navier-Stokes equations. In this paragraph we will investigate this issue. We will explain also why the rather elementary geometric calculus approach is better so as to solve the millennium problem, compared to more advanced functional analysis.

One primary point, known but often forgotten is the next. The Euler and the Navier-Stokes equations are the equations that are considered to govern the flow of fluids, and had been formulated long ago in mathematical physics before it was known that matter consists from atoms. So actually they formulated the old infinite divisible material fluids. After L. Boltzmann and the discovery of material atoms, the truer model is that of statistical mechanics. We may consider that the two different types of matter, a) infinite divisible b) made from finite atoms, behave the same as far as flows in fluid dynamics, and certainly there are many common properties but ultimately are mathematically and logically different. One example of the difference is that in the atomic structured material fluid model, the angular velocity of the spin e.g. of electrons, protons, neutrons which is about 1 terahertz (infrared range) can vary increase or decrease, independently from the vorticity, which is only the part of the angular velocity which is "geared to the environmental" rotation of the fluid. In the classical Weierstrass calculus of infinite divisible material fluids (Euler and Navier-Stokes equations) this distinction does not exist and all the angular velocity of a point is due to the vorticity. In [35] Muriel, A. 2000 a corresponding to the millennium problem in statistical mechanics has been solved in the direction of regularity. Similarly, in [27] Kyritsis, K. November 2017 a solution of the current millennium problem has been proved in the direction of regularity, but only if adding an additional hypothesis to the initial formulation, that of existence of finite atomic particles that are conserved during the flow. Strictly speaking a mathematical model of the material fluids and their flow which will have a high degree of exactness should take in to account that matter consists of atoms, (the electron range of magnitudes is of the order $10^{-15}$ meters) and this suggests that we should avoid utilizing concepts of continuity and smoothness that use $\varepsilon>0 \delta>0$ in their definition smaller than $10^{-15}$ meters. To address this difficulty of our current (Weierstrass) calculus the author developed the Democritus digital and finite decimal differential calculus (see [30] Kyritsis K. 2019 ,[31]Kyritsis K. 2017 , [32] Kyritsis K. 2022) In this finite calculus, we define concepts, of seemingly infinitesimal numbers (they are finite), seemingly infinite numbers (they are finite) and feasible finite numbers, so as to develop a differential and integral calculus up to decimal numbers with only a fixed finite number of decimals (decimal density of level of precision). Different levels of precision give different definitions of continuity and smoothness. These multi-precision levels Democritus calculi is what an applied mathematician is doing when applying the Newton-Leibniz and Weierstrass calculus with the infinite (and infinitesimals). The Democritus calculus strictly speaking is not logically equivalent to the Newton-Leibniz calculus or to the Weierstrass calculus. E.g. classical Weisstrass calculus continuity corresponds in the Democritus calculus of being continuous not only to a single precision level but to all possible precision levels. Because in the Democritus calculus continuity and smoothness is only up to a precision level, the turbulence can be defined in a way that in Weierstrass calculus cannot be defined. In a turbulent flow, the flow in the Democritus calculus may be smooth relative to a precision level but non-smooth relative to a coarser precision level (or the opposite) in the Weierstrass calculus this is impossible. Furthermore, now when a computer scientist is experimenting with computers to discover if in a flow there will be a blow up or not in finite time, within the Democritus calculus and its Navier-Stokes equations he will have an absolute proof and criterion. If the vorticity will become seemingly infinite (still finite) in a feasible finite time interval there is a blow up. If it becomes only feasible finite in any feasible finite time interval, there is no blow up. Of course blow-up in the Democritus calculus is not equivalent with a blow up in the Weisstrass calculus. Finally, with the Democritus calculus the applied 
mathematician acquires the subjective quality of congruence. In other words, what he thinks, sais and writes is what he acts and applies. With the infinite in the ontology of calculus this is not possible and it is unavoidable the incongruence, because infinite cannot be acted in the applications in a material reality where all are finite.

It is known that when the calculus (which is used in modelling the fluids) was discovered by Newton and Leibniz, the original mathematical ontology was utilizing infinitesimals, smaller than any positive real numbers but not zero. Then later with Weierstrass calculus this ontology was abandoned, we restricted ourselves to the real numbers only, and we utilized limits and convergence. So when we take the law of force (momentum conservation) of Newton $\mathrm{F}=\mathrm{m}^{*} \gamma$ on a solid finite particle and then take the limit by shrinking it to a point to derive the Euler and Navier-Stokes equations, we must not forget, that originally the limit was not to a point but to an infinitesimal solid body particle. This is not the same! In [32] Kyritsis K. 2022, I have restored with strict mathematics the original ontology of infinitesimals of Newton-Leibniz, utilizing algebra of intervals (or inverses of ordinal numbers as J. H Conway has also done with the surreal numbers see[9] J H. Conway and [33] K Kyritsis ordinal real numbers 1,2,3). Then we have a two-density calculus with two different linearly ordered fields, a) the real numbers b) a larger such field of Newton-Leibniz fluxions, with infinitesimal, finite and infinite numbers. The topologies of convergence of a solid finite particle by shrinking it to a point or to an infinitesimal solid particle are different! And this affects the issue of vorticity and angular velocity of infinitesimal particle. When I was a University student, and I was learning about the equations of Navier-Stokes, I was satisfied to see that the simple law of force (momentum conservation) of Newton $\mathrm{F}=\mathrm{m}^{*} \gamma$ was converted to the Navier-Stokes equations, but I was shocked to realize, that the rest of the independent information about the motion of the solid finite particle, namely its rotational momentum, was not shrunk to an angular velocity $\omega$ of the infinitesimal solid particle. So we see now that this is not reasonable in the Weisstrass calculus, which shrinks to a point, while it is possible in the older Newton-Leibniz calculus which shrinks to an infinitesimal solid body, and would lead to a different model of flows of fluids, with independent initial data of angular velocities, besides linear velocities and besides the derived from them vorticity.

In the current solution of the millennium problem, we may observe a $20 \%-80 \%$ Pareto rule. In other words, more than $80 \%$ of the equations utilized as governing equations of the flow, are those derived from fundamental theorem of the calculus, (in the form of Stokes theorem, divergence theorem, green theorem, Helmholtz-kelvin theorem, fundamental theorem of calculus etc.) and less that $30 \%$ the PDE of the Navier-Stokes equations. So I might say that the main equations governing the phenomenon of flow is the machinery of exterior differential algebra (wedge product) differentiation (differential forms) etc.rather than simply PDE equations. For reasons of simplicity and because we are restricted here to only 3 spatial dimensions, we do not utilize the symbolism of the wedge products and differential forms, but only the Stokes theorem, divergence theorem etc.
These versions of the fundamental theorem of the calculus (Stokes theorem etc.) lead to an extension of the law of momentum conservation of 3D fluid parts to a law of 1D line density (rotatory) momentum conservation (Theorem 3.1) and law of 2D surface density (rotatory) momentum conservation (Theorem 3.2). These laws are very valuable for infinite divisible fluids so valuable as the existence of finite atoms in the atomics structured fluids. Without these extra laws of momentum density conservation, we would have no hope to solve the millennium problem. As T. Tao had remarked, only an integral of 3D energy conservation and an integral of $3 \mathrm{D}$ momentum conservation is not adequate to derive that momentum point densities $\rho \bullet \mathbf{u}$, or energy point densities $(1 / 2) \rho \bullet u^{2}$ will not blow up.

Besides the forgotten conservation law of finite particles, which unfortunately we cannot utilize in the case of infinite divisible fluids to solve the millennium problem, there are two more forgotten laws of conservation or invariants The first of them is the obvious that during the flow, the physical measuring units dimensions (dimensional analysis) of the involved physical quantities (mass density, velocity, vorticity, momentum, energy, force point density, pressure, etc.) are conserved. It is not very wise to eliminate the physical magnitudes interpretation and their dimensional analysis when trying to solve the millennium problem, because the dimensional analysis is a very simple and powerful interlink of the involved quantities and leads with the physical interpretation, to a transcendental shortcut to symbolic calculations. By eliminating the dimensional analysis, we lose part of the map to reach our goal.

The $2^{\text {nd }}$ forgotten conservation law or invariant, is related to the viscosity (friction). Because we do know that at each point (pointwise), the viscosity is only subtracting kinetic energy, with an irreversible way, and converting it to thermal energy, (negative energy point density), and this is preserved in the flow, (it can never convert thermal energy to macroscopic kinetic energy), we know that its sign does not change too, it is a flow invariant, so the integrated $1 \mathrm{D}$ or $2 \mathrm{D}$ work density is always of the same sign (negative) and as sign, an invariant of the flow. The conservation or invariance of the sign of work density by the viscosity (friction) is summarized in the lemma 3.1 below.

Finally we must not understate the elementary fact that the force densities $\mathrm{F}_{\mathrm{p}}$ due to the pressures $\mathrm{p}$,

$F_{p}=-\nabla p \quad$ are conservative, irrotational vector field, and they do not contribute to the increase or decrease of the rotatory momentum and vorticity of the fluid during the flow. Because of this we get that the conserved 1D and 2D densities of momentum in Theorems 3.1 and 3.2 are only of the rotatory type.

Anyone who has spent time to try to prove existence of Blow up or regularity in the various physical quantities of the fluid like velocity, vorticity, acceleration, force density, momentum, angular momentum, energy etc. he will observe that in the arguments the regularity and uniform in time boundedness propagates easily from derivatives to lower order of differentiation, while the blowup arguments propagate easily from the magnitudes to their derivatives. The converses are hard in proving. This is due to the usual properties of the calculus derivatives and integrals. The hard part of the proofs, must utilize forms of the fundamental theorem of the calculus like Stokes theorem, divergence theorem etc. 
Based on the above remarks about what is not very well understood with Navier-Stokes equations I decided that elementary geometric calculus should be the appropriate context to solve the millennium problem, and this I did indeed.

Here in the next we apply the idea that the most valuable equations that govern he flow of the fluid are not literally the Navier-Stokes equations but the invariants or semi-invariant properties of the flow, derived from the abstract multi-dimensional fundamental theorems of calculus, in the forms of divergence theorems, Stokes theorems, Greens theorems etc. Actually this is the mechanism of wedge-products and abstract algebra of differential forms which is beyond classical partial differential equations. We do not utilize though definitions and symbolism of wedge-products and differential forms in his paper so as to keep it elementary and easy to read. The main discovery of this paragraph is the Helmholtz-Kelvin-Stokes theorem 3.3 in the case of viscous flows and the resulting general no-blow-up theorem 3.4 for the viscous flows without external forcing. A blow-up when it occurs, it will occur at least as blow-up of the vorticity, or of $\rho^{\bullet} \omega$. If we discover average value invariants of the flow with physical units dimensions $\rho \bullet \omega$, that in the limit can give also the point value of the $\rho \bullet \omega$, and that are invariants independent from the size of averaging, it is reasonable that we can deduce conclusions, if the point densities can blow-up or not.

Theorem 3.1 The Helmholtz-Kelvin-Stokes theorem in the case of inviscid Euler equations flows without external force or homogeneous case. (A1D line density of rotatory momentum, conservation law).

Let initial data in $R^{3}$ so that they guarantee the existence of a unique smooth solution to the Euler equation in a local time interval $[0, T]$. Then at any time $t \in[0, T]$ the circulation $\Gamma(c)$ of the velocities on a closed smooth loop is equal to the flux of the vorticity on smooth surface $S$ with boundary the loop $c$, and is constant and preserved as both loop and surface flow with the fluid. In symbols $(\rho=1$ is the density of the incompressible fluid)

$\Gamma_{c(t)}=\rho \oint_{c=\partial S} u d l=\rho \iint_{S} \omega \bullet d s(e q .3 .1)$

Proof: See[34] Majda, A.J-Bertozzi, A. L. 2002, Proposition 1.11 and Corollary 1.3 , in page 23. The proof is carried actually by integrating the Euler equations on a loop $\mathrm{c}$ and utilizing that the integral of the pressure forces (densities) defined as $-\nabla p$ are zero as it is a conservative (irrotational) field of force (densities). Then by applying also the Stokes theorem that makes the circulation of the velocity on a loop equal to the flux of the vorticity on a smooth surface with boundary the loop (see e.g. Wikipedia Stokes theorem https://en.wikipedia.org/wiki/Stokes\%27_theorem)the claim is obtained. QED.

We may notice that this circulation and surface vorticity flux has physical measuring units $[\rho]^{*}[\omega]^{*}[\mathrm{~s}]^{\wedge} 2=[\mathrm{m}]^{*}[\mathrm{~s}]^{\wedge}(-3)^{*}[\mathrm{t}]^{\wedge}(-1)[\mathrm{s}]^{\wedge} 2=[\mathrm{m}]^{*}[\mathrm{~s}]^{\wedge}(-1)^{*}[\mathrm{t}]^{\wedge}($ $-1)=[\text { moment_of_inertia }]^{*}[\omega]^{*}[\mathrm{~s}]^{\wedge}(-3)$ thus angular momentum point density. While the $\rho^{*} \omega$ has physical measuring units dimensions $[\rho]^{*}[\omega]=[\mathrm{m}]^{*}[\mathrm{~s}]^{\wedge}(-3]^{*}[\mathrm{t}]^{\wedge}(-1)$ $=[\text { moment_of_inertia }]^{*}[\omega]^{*}[\mathrm{~s}]^{\wedge}(-2)$ thus $2^{\text {nd }}$ spatial derivative of rotational momentum of point density .

A blow-up when it occurs, it will occur at least as blow-up of the vorticity, or of $\rho \bullet \omega$. If we discover bounded average value invariants of the flow with physical units dimensions $\rho \bullet \omega$, that in the limit can give also the point value of the $\rho^{\bullet} \omega$, and that are invariants and bounded independent from the size of averaging, it is reasonable that we can deduce conclusions, if the point densities can blow-up or not.

Here we convert the surface vorticity flux invariant of Helmholtz-Kelvin-Stokes to one with 3D integration which will be more convenient in the arguments as the volumes are preserved by incompressible flows and most important, the integration is 3-dimensional which can be utilized to define average values of the vorticity (flux) on 3D finite particles..

We will prove at first a lemma about the 3D volume integral of Theorem 3.2, and convergence of average values of vorticity, based on this $3 \mathrm{D}$ integral, to point values to vorticity.

We define an average value for the volume 3D integral of vorticity flux.

Definition 3.1 We define as average value on ball in of the vorticity $\omega$, denoted by $\bar{\omega}_{B}$, the unique constant value of the vorticity on the interior of the ball that would give the same $3 D$ flux of vorticity on the ball, $\rho \int_{0}^{\pi} \iint_{S} \bar{\omega} \cdot d s d \theta=$ $\rho \int_{0}^{\pi} \iint_{S} \omega \bullet d s d \theta$.The integration on the surfaces $S$ for the flux of the vorticity is on parallel circular discs in the ball. This average value $\bar{\omega}$ of the vorticity is of course the

$\left\|\bar{\omega}_{B}\right\|=\left|\frac{\rho \int_{0}^{\pi} \iint_{S} \omega \bullet d s d \theta}{|B|}\right|(e q .3 .2)$

and its direction is that of the vertical axis of the ball $B$

Where $|B|=(4 / 3) * \pi * r^{3}$ is the volume of the ball $B$, of radius $r$, and $\left\|\omega_{B}\right\|$ is the Euclidean norm of the vector. A more detailed symbolism of the average vorticity is the $\bar{\omega}\left(x_{t}, t\right)_{B(r, t)}$

The numerator of this average value of vorticity has also the interpretation of rotational momentum average axial density on the ball $B$ and relative to the axis a. A reason for this is that the physical dimensions of measuring units of this magnitude is that of rotational momentum line density. This is because the rotational momentum point density has physical

dimensions [moment_of_inertia] ${ }^{*}[\omega]^{*}[s]^{\wedge}(-3)=[m][s]^{\wedge}(-1)[t]^{\wedge}(-1)$, where $[\bar{m}]$ for mass, $[s]$ for distance, [t] for time, and this magnitude has physical units dimensions, $\left([\rho][\omega][s]^{\wedge} 3\right.$ )$=\left([m][s]^{\wedge}(-1)[t]^{\wedge}(-1)\right)[s]^{\wedge}(1), \quad$ thus rotational momentum point density integrated on 1-d line axial density. And the full 
quotient therefore has physical units dimensions $\left.[m][s]^{\wedge}(-3)[t]^{\wedge}(-1)\right)=[\rho][\omega]$.

Lemma 3.1 Let a ball $B$ of radius $r$ and center $x$, and the average vorticity $\bar{\omega}_{B}$ in it as in the Definition 4.1 so that its axis a that defines the average vorticity is also the axis of the point vorticity $\omega_{x}$ at the center $x$ of the ball. By taking the limit of shrinking the ball to its center $x,(r->0)$, the average vorticity $\bar{\omega}_{B}$ converges to the point vorticity $\omega_{x}$. In symbols $\lim _{r \rightarrow 0} \bar{\omega}_{B}=\omega_{x}$. If the axis a of the ball to estimate the average vorticity is not the axis of the point vorticity, then the limit of the average vorticity will be equal to the projection component $\omega_{\mathrm{a}}(\mathrm{x}, \mathrm{t})$ of the point vorticity $\omega(\mathrm{x}, \mathrm{t})$ on the axis a.

Proof: We simply apply an appropriate 3-dimensional version, with iterated integrals of the 1-dimensional fundamental theorem of the calculus. QED.

Remark 3.1. Such a limit of $3 \mathrm{D}$ body to a point is the same as the limit that from the Newton equation of force $\mathrm{F}=\mathrm{m} \gamma$, we derive the Navier-Stokes equations.

Since the flow of a fluid under the Euler or Navier-Stokes equations, with or without smooth Schwartz external force is a smooth and continuous mapping $\mathrm{F}$, then such a limit will be conserved to still be a valid limit during the flow. In other

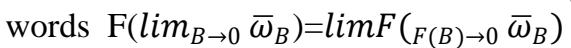

and $\mathrm{B}->0$, implies $\mathrm{F}_{\mathrm{t}}(\mathrm{B})->0$. We define of course in an obvious appropriate way the average vorticity $\mathrm{F}_{\mathrm{t}}\left(\bar{\omega}_{B}\right)$ as in definition 3.1, for the flow-image of a ball B after time $t$. Simply the disc surfaces will no longer be flat, and the loop no longer perfect circle. But the integrals in the definition will be the same. Constancy of the average vorticity on such surfaces will only be, up to its Euclidean norm and vertical angle to the surface. We must notice though that although a relation $\mathrm{F}\left(\lim _{B \rightarrow 0} \bar{\omega}_{B}\right)=\lim F\left({ }_{B \rightarrow 0} \bar{\omega}_{B}\right)$ would hold, the value of this limit will not be the vorticity $\omega_{\mathrm{F}(\mathrm{x})}$ at the flowed point! Unfortunately, the Lemma 3.2 holds not on arbitrary 3D shapes and arbitrary integration parametrization on it, but only when we start with standard 3D shapes like a sphere, a cylinder a cube etc. and the normal parametrization on them. The reason is that we need to take in to account in a normal way the average vorticity around a point in an unbiased way, that an arbitrary shape will not give.

Another important conservation point is that the relation of the vorticity $\omega_{\mathrm{x}}$ being tangent to an axis a (or general curve) is conserved during inviscid Euler flows. It is the conservation of vorticity lines (See [34] Majda, A. J. -Bertozzi, A. L. 2002, Proposition 1.9 in page 21). Therefore for inviscid (and incompressible) flows the axis of the initial point vorticity $\omega(0)$, which is also the axis to estimate the average vorticity on the ball B, will still be after the flow and at time t, tangent to the point vorticity $\omega(\mathrm{t})$. But for general viscous flows this will not be so. Notice that such limits of average values would not work for the circulation of the velocity on a loop, as in the application of the iterated 1-dimensional fundamental theorem of the calculus would require boundaries of the integration.
Lemma 3.2 Let the Euler or Navier-Stokes equations of incompressible fluids in the non-periodic or periodic setting, with smooth initial data and we assume that the initial data in the periodic or non-periodic case, are so that the supremum of the vorticity is finite denoted by $F_{\omega}$ on all 3-space at time $t=0$. Let the average vorticity, or average rotational momentum density, defined as in Definition 4.1 but with integration parametrization one any smooth $3 D$ shape $B$ of any size, that of course both as a diffeomorphic image of a spherical ball with its spherical coordinates integration parametrization. Then the average vorticity or average rotational momentum density is also upper bounded by the $F_{\omega .}$ In symbols

$$
\left\|\bar{\omega}_{B}\right\|=\left|\frac{\int_{0}^{\pi} \iint_{S} \omega \bullet d s d \theta}{|B|}\right| \leq \mathrm{F}_{\omega}(\text { eq. 3.3) }
$$

Proof: Since $\|\omega\|<=\mathrm{F}_{\omega}=\|(\omega /\|\omega\|)\| \mathrm{F}_{\omega}$ in the flux-integration we have for the inner product of $\omega$ and the unit area vector $n$, $(\omega, \mathrm{n})<=\left((\omega /\|\omega\|) \mathrm{F}_{\omega}, \mathrm{n}\right)<=\mathrm{F}_{\omega}$. Thus in the integration we may factor out the $F_{\omega}$

$$
\begin{aligned}
& \left|\frac{\int_{0}^{\pi} \iint_{S} \omega \cdot d s d \theta}{|B|}\right| \leq\left|\frac{\int_{0}^{\pi} \iint_{S} F_{\omega} d s d \theta}{|B|}\right|=\mathrm{F}_{\omega}\left|\frac{\int_{0}^{\pi} \iint_{S} \mathrm{ds} d \theta}{|\mathrm{B}|}\right|=\quad \mathrm{F}_{\omega}\left|\frac{|B|}{|\mathrm{B}|}\right|= \\
& \mathrm{F}_{\omega} \cdot \mathrm{QED} .
\end{aligned}
$$

\section{Lemma 3.3 The viscosity sign forgotten invariant.}

If we integrate the force point density of the viscosity, over a line (1D work density) or surface (2D work density) or a volume (work) its sign will remain the same during the flow.

Proof:Because we do know that pointwise, the viscosity is only subtracting kinetic energy, with an irreversible way, and converting it to thermal energy, (negative energy point density), and this is preserved in the flow, (it can never convert thermal energy to macroscopic kinetic energy), we deduce that its sign does not change too it is a flow invariant, so the integrated $1 \mathrm{D}$ or 2D work density is always of the same sign (negative) and as sign an invariant of the flow. QED.

Theorem 3.2 A 3-dimensionl integral version of the Helmholtz-Kelvin-Stokes theorem. (A2D surface density of rotatory momentum, conservation law).

Let initial data in $R^{3}$ so that they guarantee the existence of a unique smooth solution to the Euler equation in a local time interval $[0, T]$. Then at any time $t \in[0, T]$ let a sphere $B$ of radius $r$ (as in figure 4.) considered as a finite particle, then the azimuthal $\theta$-angle, $\theta$-integral on a meridian in spherical coordinates of the circulations $\Gamma(c)$ of the velocities on all closed longitude smooth loops parallel to the equatorial loop is equal to the same $\theta$-integral of the surface flux of the vorticity on smooth flat disc surfaces $S$ with boundary the loops c (as in figures 4.2), and both integrals are constant and preserved as both surface and volume integrals during the flow with the fluid. In symbols $(\rho=1$ is the density of the incompressible fluid)

$\rho \int_{0}^{\pi} \oint_{c=\partial S} u d l d \theta=\rho \int_{0}^{\pi} \iint_{S} \omega \cdot d s d \theta(e q .3 .4)$ 
After (eq. 3.2) $\| \bar{\omega}_{B}||=\left|\frac{\int_{0}^{\pi} \iint_{S} \omega \bullet d s d \theta}{|B|}\right|$ it holds also

for $t \in[0, T]$

$\overline{\| \omega}_{B(0)}\|=\| \bar{\omega}_{B(t)} \|($ eq. 3.5)

Proof: We simply take the $\theta$-azimuthal angle $\theta$-integral of both sides of the equation 3.1 in the theorem 3.1. Both sides are preserved during the flow and so is their $\theta$-integrals too.

We notice that the measuring physical units dimensions

of the conserved quantity $\rho \int_{0}^{\pi} \oint_{c=\partial S} u d l d \theta$ is $[\text { mass }]^{*}[\text { length }]^{\wedge}(-3)^{*}[\text { velocity }]^{*}[\text { length }]^{\wedge}(2)=$ [mass $]^{*}[\text { length }]^{\wedge}(-2)^{*}[$ velocity] thus integration in 2-dimension surface of momentum 3D-point-density, or equivalently momentum 1D density QED

Theorem 3.3. The Helmholtz-Kelvin-Stokes theorem in the case of viscous Navier-Stokes equations flows without external force (homogeneous case).

Let initial data in $R^{3}$ so that they guarantee the existence of a unique smooth solution to the Navier-Stokes equation with viscosity coefficient $v>0$, in a local time interval $[0, T]$. Then at any time $t \in[0, T]$ the circulation $\Gamma(c)$ of the velocities on a closed smooth loop is equal to the flux of the vorticity on smooth surface $S$ with boundary the loop $c$, and is decreasing as both loop and surface flow with the fluid. In symbols $(\rho=1$ is the density of the incompressible fluid)

$\rho \oint_{c=\partial S} u d l=\rho \iint_{S} \omega \bullet d s(e q .3 .1)$

And for $t \in[0, T]$

$\oint_{c=\partial S} u(0) d l>\oint_{c=\partial S} u(t) d l($ eq. 3.6)

and similarly for the $3 D$ volume integration as in Theorem 3.2

for $t \in[0, T]$

$\rho \int_{0}^{\pi} \iint_{S} \omega(0) \cdot d s d \theta>\rho \int_{0}^{\pi} \iint_{S} \omega(t) \bullet d s d \theta($ eq. 3.7)

After (eq. 3.2) $\| \bar{\omega}_{B}||=\left|\frac{\int_{0}^{\pi} \iint_{S} \omega \bullet d s d \theta}{|B|}\right|$ it holds also for initial finite spherical particles for $t \in[0, T]$

$\left\|\bar{\omega}_{B(0)}\right\|>\left\|\bar{\omega}_{B(t)}\right\|($ eq. 3.8)

Proof: AgainThe (eq. 3.1)is nothing else of course but the Stokes theorem.
We shall utilize here the next equation (See[34] Majda, A.J-Bertozzi, A. L. 2002, (eq 1.61), in page 23.) in the case of viscous incompressible flows under the Navier-Stokes equations

$\frac{d}{d t} \Gamma_{c(t)}=\frac{d}{d t} \oint_{c(t)} u d l=v \oint_{c(t)} \Delta u d l=-v \oint_{c(t)} c u r l \omega d l,(e q$. 3.9)

This equation is derived after applying as in Theorem 3.1 the loop integral of the circulation at the Navier-Stokes equations instead at the Euler equations taking the material-flow derivative outside the integral, and eliminating the conservative, irrotational part of the pressure forces as gradient of the pressure. Here the viscosity is not zero thus the left hand of the equations is not zero as in the case of Euler equations, where it is conserved. The right hand side is nothing else than the loop work density of the point density of the force of viscosity at any time $t$. And as the viscosity always subtracts energy, this right hand side work density is always negative during the flow. We notice after the Lemma 3.3that the viscosity force point density keeps constant sign on the trajectory path as orbital component during the flow and relative to the velocity on the trajectory. It is always as orbital component opposite to the motion and represents the always irreversible energy absorption and linear momentum and angular momentum decrease. Similarly, for any rotation of the fluid e.g. with axis the trajectory path. The viscosity force point density as component on the loop is always opposite to the rotation, it never converts thermal energy to add to linear or angular momentum. This opposite to motion monotonicity of the viscosity force density applies to the Navier-Stokes equations but also as opposite to rotation monotonicity in the vorticity equation $\frac{D \omega}{D t}=\omega * \nabla \mathrm{u}+v \Delta \omega$ (see [34] Majda, A.J-Bertozzi, A. L. 2002, (eq 1.33 ) and (eq 1.50 ) in pages 13 and 20 ). So if we choose a direction of the loop so that the circulation integral on the right hand side is positive then this will have the same sign during the flow (although different absolute value), and will make the left hand side of the (eq. 4.9) always negative during the flow.But this means from the left-hand side of the equation that the circulation of the velocity on the loop is always decreasing during the flow.

$\frac{d}{d t} \oint_{c(t)} u d l<0 \quad$ for any $\mathrm{t}$ in $[0, \mathrm{~T}] \quad$ (eq. 3.10)

Thus(eq. 3.6) is proved, and (eq. 3.7) is direct consequence.

To prove the equation 3.8 we notice that due to incompressibility, the flow is volume preserving, thus $|\mathrm{B}(\mathrm{x}(\mathrm{t}))|=\mid \mathrm{B}(\mathrm{x}(0) \mid$, and by dividing both sides of the equation 4.7 , and after the definition

$\left\|\bar{\omega}_{B}\right\|=\left|\frac{\int_{0}^{\pi} \iint_{S} \omega \bullet d s d \theta}{|B|}\right|$

it holds also

for $\mathrm{t} \in[0, \mathrm{~T}]$

$\left\|\bar{\omega}_{\mathrm{B}(0)}\right\|>\left\|\bar{\omega}_{\mathrm{B}(\mathrm{t})}\right\|($ eq. 3.8)QED.

Theorem 3.4 The no blow-up theorem in finite or infinite time in the Euler, Navier-Stokes, periodic or non-periodic and homogeneous cases. 
Let the Euler or Navier-Stokes equations of incompressible fluids in the non-periodic or periodic setting (homogeneous case with no external forces), with

a) smooth initial data and whatever else hypothesis is necessary so as, also to guarantee the existence and uniqueness of smooth solutions to the equations locally in time $[0, T)$.

b) Furthermore we assume that the initial data in the periodic or non-periodic case, are such that the supremum of the vorticity, denoted by $F_{\omega}$, is finiteat $\mathrm{t}=0$. (In the periodic case, smoothness of the initial velocities is adequate to derive it, while in the non-periodic setting smooth Schwartz initial velocities is adequate to derive it)

Then it holds that there cannot exist any finite or infinite time blow-up at the point vorticities during the flow.

Proof: The proof will by contradiction. The main idea of the proof is to utilize that in the case of a blow-up the vorticity will converge to infinite, so it will become larger than an arbitrary lower bound $\mathrm{M}+\mathrm{F}_{\omega}, \mathrm{M}>0, \mathrm{~F}_{\omega}>0$ and by approximating it with average flux vorticity of a 3D spherical particle, and tracing it back at the initial conditions where all is bounded by $\mathrm{F}_{\omega}$, utilizing the semi-invariance of the average vorticity that we have proved, we will get that $\mathrm{F}_{\omega}>\mathrm{M}+\mathrm{F}_{\omega}$.

So let us assume that there is a blow up, in a finite time or infinite time $\mathrm{T}^{*}$, with the hypotheses of the theorem 4.2. Then from the Theorem 2.2 and (eq. 2.12) which is the well-known result of the control of regularity or blow up by the vorticity we get that,

$\lim _{\mathrm{t} \rightarrow \mathrm{T} *} \int_{0}^{\mathrm{T}}|\omega(., \tau)|_{\mathrm{L}^{\infty}} \mathrm{d} \tau=+\infty($ eq. 2.12)

We conclude that there will exist an infinite sequence of points $\left\{\mathrm{x}_{\mathrm{tn}}, \mathrm{n}\right.$ natural number, $\left.0<\mathrm{t}_{\mathrm{n}}<\mathrm{T}^{*}, \lim _{\mathrm{n} \rightarrow \infty} t_{n}=T^{*}\right\}$ so that the point vorticity $\omega\left(x_{t n}\right)$ blows-up, or equivalently $\lim _{n \rightarrow \infty} \omega\left(x_{t n}\right)=+\infty$. We do not need to assume them on the same trajectory. Therefore, for every positive arbitrary large real number $\mathrm{M}_{0}$, there is a $\mathrm{n}_{0}$ such that for all natural numbers $\mathrm{n}>\mathrm{n}_{0}$, it holds that $\omega\left(\mathrm{x}_{\mathrm{tn}}\right)>\mathrm{M}_{0}$. We choose $\mathrm{M}_{0}=\mathrm{M}_{00}$ $+\mathrm{F}_{\omega}$, for an arbitrary large positive number $\mathrm{M}_{00}$. So

$\omega\left(\mathrm{x}_{\mathrm{tn}}\right)>\mathrm{M}_{00}+\mathrm{F}_{\omega}$ (eq. 3.11)

Now we approximate this point vorticity with an average flux vorticity on a 3D particle after Definition 3.1 , theorem 3.2 and Lemma 3.1.

Let a spherical ball particle $\mathrm{B}\left(\mathrm{r}, \mathrm{x}_{\mathrm{tn}},\right)$ as in theorem 3.2. with center $x_{t n}$ and radius $r>0$. After Definition 3.1 , theorem 3.2 and Lemma 3.1. we have that

$\lim _{\mathrm{r} \rightarrow 0} \bar{\omega}_{\mathrm{B}}=\omega_{\mathrm{x}(\mathrm{tn})}$, With $\overline{\| \omega}_{\mathrm{B}}||=\left|\frac{\int_{0}^{2 \pi} \iint_{\mathrm{S}} \omega \cdot \mathrm{ds} d \theta}{|\mathrm{B}(\mathrm{r}, \mathrm{x}(\mathrm{tn}))|}\right|$

Therefore for arbitrary small positive number $\varepsilon>0$, there is radius $R$, with

$$
\bar{\omega}_{B(R)}>\omega_{x(t n)}-\varepsilon
$$

$\operatorname{or}\left|\frac{\int_{0}^{2 \pi} \iint_{S} \omega \bullet d s d \theta}{|\mathrm{B}(\mathrm{r}, \mathrm{x}(\mathrm{tn}))|}\right|>\omega_{x(t n)}-\varepsilon($ eq. 3.12)

Thus after (eq. 3.11)

$\left|\frac{\int_{0}^{2 \pi} \iint_{S} \omega \bullet d s d \theta}{|\mathrm{B}(\mathrm{R}, \mathrm{x}(\mathrm{tn}))|}\right|>M_{00}+F_{\omega}-\varepsilon($ eq. 3.13)

Now we trace back on the trajectory of the $x_{t n}$ the parts of the (eq. 3.13)

At initial time $\mathrm{t}=0$. We use the advantage that as the incompressible flow is volume preserving, the $\left|\mathrm{B}\left(\mathrm{R}, \mathrm{x}_{0},\right)\right|=$ $|\mathrm{B}(\mathrm{R}, \mathrm{x}(\mathrm{tn}))|$. We also utilize theorems 3.2, 3.3, and (eq. 3.5 ), (eq. 3.8), which prove that at the initial conditions $\mathrm{t}=0$, this average vorticity is the same or higher than that at $t_{n}$.

$$
\left|\frac{\int_{0}^{2 \pi} \iint_{S} \omega \bullet d s d \theta}{|\mathrm{B}(\mathrm{R}, \mathrm{x}(0))|}\right| \geq\left|\frac{\int_{0}^{2 \pi} \iint_{S} \omega \bullet d s d \theta}{|\mathrm{B}(\mathrm{R}, \mathrm{x}(\mathrm{tn}))|}\right|
$$

We conclude that

$$
\left|\frac{\int_{0}^{2 \pi} \iint_{S} \omega \bullet d s d \theta}{|\mathrm{B}(\mathrm{R}, \mathrm{x}(0))|}\right|>M_{00}+F_{\omega}-\varepsilon(\text { eq. 3.14) }
$$

From the (eq. 3.14) and (eq. 3.3) of Lemma 3.2 we conclude that

$$
\mathrm{F}_{\omega}>M_{00}+F_{\omega}-\varepsilon
$$

But $\mathrm{M}_{00}$ was chosen in an independent way from $\varepsilon>0$ to be arbitrary large, while $\varepsilon>0$ can be chosen to be arbitrary small. Therefore, a contradiction. Thus there cannot be any blow-up either in finite or infinite time $\mathrm{T}^{*}$. QED.

Remark 3.2. Infinite initial energy. We must remark that we did not utilize anywhere that the initial energy was finite, only that the vorticity initially has finite supremum. Thus this result of no-blow-up can be with infinite initial energy too. But when applying it to the millennium problem we do have there also that the initial energy is finite.

Remark 3.3. A strange blow up for any time $t>0$ of initially smooth data. We might be curious to ask the question if it is possible, starting with zero initial velocities and pressures, to create an artificial blow-up only with external forcing. A good candidate is the perfect circular vortex, where all the trajectory paths are perfect circles, 
which is known that it is an instance of the solution of the Euler and Navier-Stokes equations. We can formulate the circular vortex in 3D with cylindrical or spherical coordinates. But for simplicity we will formulate it in 2 dimensions, in spite the fact that we do know that in 2D dimensions there cannot be a blow up under the hypotheses of the millennium problem. So with an external forcing also as perfect circular vortex that in polar coordinates are as follows

$\mathrm{F}_{\mathrm{r}=0,}, \mathrm{~F}_{\theta}=2 \rho /(1+\exp (\mathrm{r}))$

we raise the absolute initial rest within finite time $t$ the flow to a circular vortex which has velocities in polar coordinates

$\mathrm{u}_{\mathrm{r}=0,}, \mathrm{u}_{\theta}=2 \mathrm{t} /(1+\exp (\mathrm{r}))(\mathrm{eq} 3.17)$

Now it is elementary to show that

1) this flow follows the Euler and Navier-Stokes equations

2) Because curl $\omega=0$, the viscosity has no effect it is as if an inviscid flow.

If $\omega$ is the vorticity then it is calculated in polar coordinates at the verical $\mathrm{z}$-axis by the formula

$\omega_{z}=\frac{u_{\theta}}{r}+\frac{\partial u_{\theta}}{\partial r}-\frac{\partial u_{r}}{r \partial r}$ (eq. 3.18)

4) Although the velocity has smooth polar coordinates, the vorticity is in steady blow-up (singularity) at $r=0$ for any $t>0$. That is although at $\mathrm{t}=0$ the initial data are smooth, for any $\mathrm{t}>0$, there is a blow-up.

5) The 4) is so because the external forcing although it has smooth polar coordinates, in the Cartesian coordinates, it has $\operatorname{curl}(\mathrm{F})=+\infty$, at $\mathrm{r}=0$, thus it does not satisfy the smooth Schwartz condition external forcing of the millennium problem.

\section{THE SOLUTION OF THE $4^{\mathrm{TH}}$ CLAY MILLENNIUM PROBLEM ABOUT THE NAVIER-STOKES EQUATIONSIN THE NON-PERIODIC HOMOGENEOUS CASE.}

We are now in a position to prove the Conjectures (A) and (B), non-periodic and periodic setting, homogeneous case of the Millennium problem.

(Millennium Homogeneous Case A) Existence and smoothness of Navier-Stokes solution on $\mathbf{R}^{\mathbf{3}}$.

Take $v>0$ and $n=3$. Let $u_{0}(x)$ be any smooth, divergent-free vector field satisfying (2.4). Take $f(x, t)$ to be identically zero. Then there exist smooth functions $p(x, t), u(x, t)$ on $R^{3} x[0,+\infty)$ that satisfy (2.1), (2.2), (2.3), (2.6), (2.7).

Proof: All the hypotheses of the no-blow-up theorem 4.4 are satisfied. After remark 2.4, with the current case of the millennium problem there exist indeed a unique smooth solution locally in time [0,t] (after A.J. Majda-A.L. Bertozzi

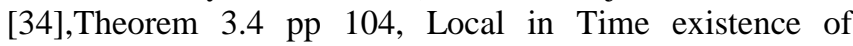
Solutions to the Euler and Navier-Stokes equations). And also the Schwartz condition of the initial data, guarantees that the supremum of the vorticity, is finiteat $t=0$. (see Remark 2.1) Therefore we conclude by Theorem 4.4 that there cannot be any finite or infinite time blow-up. Thus from Theorem 2.2 Supremum of vorticity sufficient condition of regularity we conclude that this local in time $[0, t]$ solution , can be extended in $[0,+\infty)$. QED

\section{EPILOGUE.}

In this paper it is has been proved the regularity (no blow-up) of the Navier-Stokes equations in the non-periodic setting without external forcing, and therefore it has been solved theMillennium Homogeneous Case A of the $4^{\text {th }}$ Clay Millennium problem.

\section{ACKNOWLEDGMENTS}

I would like to thank professors G. A. Athanasoulis and S. Voutsinas at the National Technical University of Athens, for their valuable encouragement and advices in fluid dynamics. I would like also to thank professor Achilles K. Tertikas Department of Mathematics \& Applied Mathematics University of Crete, for his valuable bibliographical support.

\section{REFERENCES}

[1] APOSTOL T. 1974 "Mathematical Analysis" 2nd edition Addison Wesley Publishing Company. 1974

[2] BEALE, J. T., KATO, T., AND MAJDA, A 1984., "Remarks on the breakdown of smooth solutions for the 3-D Euler equations," Commun. Math. Phys. 94, 61-66, 1984.

[3] BOGOVSKII M. E. 1980, Solution of some vector analysis problems connected with operators div and grad, Trudy Sem. S. L. Sobolev 80 (1) (1980) 5-40.

[4] CANNON, J.R., KNIGHTLY, G.H. 1970 : A note on the Cauchy problem for the Navier-Stokes equations. SIAM J. Appl. Math. 18, 641-644(1970)

[5] CHORIN A. J. 1968 Numerical solutions of the Navier-Stokes Equations Mathematics of Computation oct. 1968, vol 22, no 104 pp 745-762

[6] CONSTANTIN P. 1995, A few results and open problems regarding incompressible fluids, Notices of the AMS, 42 (1995), 658-663.

[7] CONSTANTIN P. 2001, Some open problems and research directions in the mathematical study of fluid dynamics, in Mathematics Unlimited-2001 and Beyond, Springer Verlag, Berlin, 2001, 353-360.

[8] CONSTANTIN P. 2007 On the Euler equations of incompressible fluids. Bulletin of the AMS Vol 44, no 4, October 2007, pp 603-621.

[9] CONWAY J. H. 1976 On numbers and games (Surreal numbers) Academic press 1976

[10] GATSKI T. B. GROSCH C. E.ROSE M. E.1989 The Numerical Solution of the Navier-Stokes equations for 3-Dimensional, Unsteady, Incompressible Flows by Compact Support Schemes Journal of computation Physics 82, 298-329 (1989) .

[11] GERMAIN P. 2008 Strong solutions and weak-strong uniqueness for the nonhomogeneous Navier-Stokes system Journal d'Analyse Mathématique January 2008, Volume 105, Issue 1, pp 169-196.

[12] GIGA Y. 1983 Weak and Strong Solutions of the Navier-Stokes Initial Value Problem PubL RIMS, Kyoto Univ. 19 (1983), 887-910

[13] GIGA, Y., INUI, K., MATSUI, S. 1999 : On the Cauchy problem for the Navier-Stokes equations with nondecaying initial data. Advances in fluid dynamics 4, Quad. Mat., Dept. Math., Seconda Univ. Napoli, Caserta, 27-68(1999).

[14] GIGA, Y., MAHALOV, A., NICOLAENKO, B. 2004 : The Cauchy problem for the Navier Stokes equations with spatially almost periodic initial data. Hokkaido University preprint series in mathematics 683 , (2004)

[15] GIGA, Y., MATSUI, S., SAWADA, O. 2001 : Global existence of two-dimensional Navier Stokes flow with nondecaying initial velocity. J. Math. Fluid Mech. 3, no.3, 302- 315(2001)

[16] GIGA, Y., MIYAKAWA, T.1985: Solutions in $\mathrm{Lr}$ of the Navier-Stokes initial value problem. Arch. Rational Mech. Anal. 89, no.3, 267-281(1985)

[17] GIGA Y. SAWADA, O. 2003 On regularizing-decay rate estimates for solutions to the Navier-Stokes initial value problem, Nonlinear Analysis and Applications, To V. Lakshmikantham on his 80th Birthday vol. I (2003), 549-562.

[18] HELMS L.L. (2009) Potential Theory, Universitext, Springer Verlag.

[19] FEFFERMAN C. L. 2006 Existence and smoothness of the Navier-Stokes equation, Millennium Prize Problems, Clay Math. Inst., Cambridge, MA, 2006, 57-67.

[20] FRIEDLANDER S., DENIS SERRE 2002 Handbook of Mathematical Fluid Dynamics Vol 1,2, Gulf Professional Publishing, 2002. 
[21] KATO, J. 2003 : The uniqueness of nondecaying solutions for the Navier-Stokes equations. Arch. Ration. Mech. Anal. 169, no.2, $159-175(2003)$

[22] KATO, T. 1983 : Strong L p -solutions of the Navier-Stokes equation in $\mathrm{R} \mathrm{m}$, with applications to weak solutions. Math. Z. 187, no.4, 471-480(1984)

[23] KATO, T., FUJITA, H.1962 : On the nonstationary Navier-Stokes system. Rend. Sem. Mat. Univ. Padova 32, 243-260(1962).

[24] KATO, T. PONCE, G. 1988 Commutator estimates and the Euler and Navier-Stokesequations, CPAM 41 (1988), 891-907.

[25]KYRITSIS K. 2013.Proof of the existence of global in time smooth solutions of 3-dimensional incompressible inviscid smooth flow-solutions of the Euler equations and viscous smooth flow-solutions of the Navier-Stokes equations. (The solution of the 4ths Clay millennium problem). An outline of this paper was presented in the 2nd Applied Innovation conference of TEI of Epirus in Arta 16-17 October

2013.(http://innovation-conference-epirus.blogspot.gr/2015/01/2nd-int ernational-conference-on-applied.html)http://libsearch.teiep.gr/Record/ cris-123456789-1324

[26] KYRITSIS, K. June 2017 "On the solution of the 4th Clay Millennium problem about the Navier-Stokes equations. Equivalence of smooth compact support and smooth Schwartz initial conditions". Proceedings of the 1st international conference on quantitative, social, biomedical and economic issues ICQSBEI 2017 pp 147-172

[27] KYRITSIS, K. November 2017 "On the 4th Clay Millennium problem: Proof of the regularity of the solutions of the Euler and Navier-Stokes equations, based on the conservation of particles" Journal of Scientific Research and Studies Vol 4 (11) , pp304-317, November 2017.

[28] KYRITSIS. K. Feb 2018 A solution of the 4th Clay Millennium problem about the Navier-Stokes equations. Preprint 1) http://cris.teiep.gr/jspui/handle/123456789/1628?locale=en https://www.researchgate.net/publication/323389082_A_SOLUTION _OF_THE_4_TH_CLAY_MILLENNIUM_PROBLEM_ABOUT_T HE_NAVIER-STOKES_EQUATIONS

[29] KYRITSIS, K. February 2019 "The solutions of the 3rd and 4th Clay Millennium problems" https://arxiv.org/abs/1902.07265

[30] KYRITSIS K. 2019."An introduction to the natural differential and integral calculus, without the infinite" Journal of Regional \& Socio-Economic Issues. Oct 2019, Vol. 9 Issu e 3, p70-89.

[31] KYRITSIS K.2017,An Axiomatic System for a Physical or Digital but Continuous 3-Dimensional Euclidean Geometry, Without Infinite Many Points..World Journal of Research and Review(WJRR) ISSN:2455-3956, Volume-5, Issue-4, October2017 Pages 31-43

[32] KYRITSIS K. 2022. The Newton-Leibniz number-fields of fluxions and infinitesimals and the new applied Democritus digital calculus of the $21^{\text {st }}$ century. A partial similarity of theory and practice captured as instances of the axiomatic system of abstract calculi over multi-density quantities.To appear as book in Lambert publications. See alsohttps://www.researchgate.net/publication/358425683_THE_NEW TON-LEIBNIZ_FIELDS_OF_FLUXIONS_AND_INFINITESIMAL S AND THE NEW_APPLIED_DEMOCRITUS_DIGITAL_CALC ULUS_OF_THE_21ST_CENTURY_A_partial_similarity_of_theory _and_practice

[33] KYRITSIS K. April 2017.Ordinal Real numbers 1,2,3Conference: 1st international conference on quantitative, social, biomedical and economic issues 2017 - ICQSBEI 2017 ,Athens, GreeceVolume: PROCEEDINGS pp 233-292 https://books.google.gr/books?id=BSUsDwAAQBAJ\&pg, See also https://www.researchgate.net/publication/355155715_THE_CONTIN UUM_OF_THE_SURREAL_NUMBERS_REVISITED_THE_SURR EAL_NUMBERS_DEFINED_THROUGH_TRANSFINITE_FUND AMENTAL_CAUCHY_SEQUENCES

[34] MAJDA, A.J-BERTOZZI, A. L. 2002 Vorticity and incompressible flow Cambridge University texts in applied mathematics 2002

[35] MURIEL, A 2000 An Exact Solution of the 3-D Navier-Stokes EquationDepartment of Electrical Engineering Columbia UniversityAnd Department of Philosophy Harvard University.Also A. Muriel and M. Dresden, Physica D 101, 299, 1997.

[36] POLYANIN, A. D. ZAITSEV, V.F., MOUSSIAUX 2002. Handbook of First Order Partial Differential Equations, London : Taylor\&Francis , ISBN0-415-27267-x

[37] POLYANIN, A. D 2002. Handbook of Linear Partial Differential Equations for Engineers and Scientist, Boca Raton : Chapman \&Hall /CRC press, ISBN 1-58488-299-9

[38] PRODI, G 1959 Un teorema di unicit_a per le equazioni di Navier-Stokes, Ann. Mat. Pura Appl. 48 (1959), 173-182.
[39] RUDIN, W. 1976 "Principles of Mathematical Analysis "3rd edition McGraw-Hill Inc. 1976

[40] FAKOUR M. et al 2017 Heat transfer and fluid flow of blood with nanoparticles through porous vessels in a magnetic field: A quasi-one dimensional analytical approach. Mathematical Biosciences Volume 283, January 2017, Pages 38-47

[41] SARRA, SCOTT 2003 "The method of characteristics with applications to Conservation Laws", Journal of Online mathematics and its Applications.

[42] SERRIN,J 1963 The initial value problem for the Navier-Stokes equations, In Nonlinear Problems (Proc. Sympos., Madison, Wis., pages 69\{98. Univ. of Wisconsin Press, Madison, Wis., 1963.

[43] SAWADA, O.2003 : On time-local solvability of the Navier-Stokes equations in Besov spaces. Adv. Differential Equations 8 no.4, 385-412(2003)

[44] SPIVAK, M. 1965 "Calculus on Manifolds" The Benjamin/Cumming publishing company 1965.

[45] TAO, T. 2013 Localisation and compactness properties of the Navier-Stokes global regularity problem. Analysis \& PDE 6 (2013), 25-107

[46] TAO, T. 2014 Finite time blowup for an averaged three-dimensional Navier-Stokes equation. Cornell University Library arxiv.org 